\title{
EDUCATION AND TRAINING When slavery hides in the symptoms - are we ready to see it?
}

\author{
Author: Rosie Riley ${ }^{\mathrm{A}}$
}

Intoxicated, antagonistic and with no clear reason for being in the emergency department, a patient discloses information strongly suggesting that he has been a victim of labour exploitation, the most common form of modern slavery. This significant consultation demands prioritisation and trauma-informed expertise. As well as addressing immediate healthcare needs and safety, the complex sequelae of modern slavery must be considered.

Victims are encountering healthcare services. Yet healthcare professionals don't feel equipped to recognise the signs or know what questions to ask, while key gaps within the healthcare service prevent these patients receiving the support they need.

At all levels of professional development, the practice of safeguarding is not prioritised. This is due to lack of effective training and emphasis on softer communication and safeguarding skills. Simulation training provides a safe, educational environment to build confidence and practice conducting these challenging, complex consultations. Safeguarding leads, who receive these referrals from frontline staff, should be equipped to understand the complexity of modern slavery and the strengths and weaknesses of the support services available. Finally, healthcare professionals must be involved in shaping the wider national survivorfocused response to modern slavery.

KEYWORDS: Slavery, trafficking, exploitation, emergency, safeguarding

\section{A patient in the emergency department}

One busy evening in the emergency department, my heart sank as I picked up the notes of the next patient to be seen. The 48-yearold man had spent the past few hours stumbling around the department, disgruntling staff, complaining loudly and coming close to being removed from the premises. I walked around the department, looking to see where he'd got to. He was slumped and sleeping on a chair, smelling strongly of alcohol, dishevelled and snoring. I tapped his arm and introduced myself. He followed me with a very ataxic gait, slumping instead on the couch in a bay.

Author: ${ }^{\mathrm{A}}$ junior clinical fellow in emergency medicine, NHS clinical entrepreneur, founder of VITA, Wiveliscombe, UK
His observations were stable, with no documented temperature. I started to take a history. Hearing a heavy accent, I discovered that he was from Poland, but he said he didn't need a translator. As histories go, I found this one rather difficult. We jumped all over the place, stories and symptoms that have been going on for months, sometimes years. No matter how many times I asked whether this or that was new, we delved further into untreated, ongoing chronic symptoms. There were sharp pains in the right side of his chest. Of course, it wasn't getting better! No, he hadn't been taking painkillers for it, no he doesn't see his general practitioner. He'd had pain in his left shoulder for a few months and reduced movement since a fall. Apparently, it had been X-rayed before and nothing was done. He didn't know which hospital did the X-ray, why would he? When asking whether he'd experienced specific new symptoms such as fever, coughing up blood, night sweats, he diverted back to how terrible and how untreated his chest pains and shoulder pains were, lamenting that no-one was helping him. More than once, I asked him to watch his language, highlighting that I was there to help, and to please not speak to me like that. This was always followed by a quick apology 'sorry doctor, sorry doctor', then further lamenting about his symptoms.

I think most NHS healthcare professionals reading this will recognise a patient like mine here. A patient demanding an extraordinary level of patience and compassion, difficult to muster when it's the fourth rude patient in a row, the overbearing pressure of a 6 -hour or more wait in the department, coming to the end of a long shift with only a short break to sit down and catch your breath.

I asked him what he does for work. An unusual question perhaps. He stopped, his head dropped, and he slowly told me that he had left his work the week before. He was a kitchen porter in a restaurant. 'Did you get paid for the work you did?' I asked. He looked up at me. Another unusual question. He had worked all day, every day and hadn't been paid in months. He was told he had debts to pay back, he had slept on the premises and wasn't allowed to leave. He looked angry and defeated. Eyes growing red and watery. I crouched so that we were at eye level, with one hand on his uninjured shoulder. 'Did the owner ever threaten you?' He'd started crying. He didn't respond. I decided not to go further into his memories. 'Do you feel safe right now?' He did, but with no job and no money, he had nowhere to go. He was homeless.

\section{A reflection}

As any other healthcare professional, I struggled with knowing what to do and how to respond to this information. However, the 
significance of this consultation was something I did understand. This man had disclosed information strongly suggesting that he was a victim of labour exploitation, a form of modern slavery. He was now a priority, with a potential background of complex psychological, physical and possibly sexual trauma.

Firstly, I considered his safety. There didn't seem to be any urgent threat, he was alone and had denied feeling unsafe. I still needed to examine and investigate his symptoms, ruling out emergency causes. Now however, I was looking for other clues; signs of malnourishment, skin disease, poor dental hygiene, old or delayed presentation of fractures, signs of respiratory tract infection or concerning upper lobe consolidation on his chest X-ray, suggestive of tuberculosis. Were there any neurological signs requiring a computed tomography of the head to rule out intracranial injury? I sent off all routine blood tests, including HIV.

I considered his capacity. Although intoxicated, did he have capacity to discuss and consent to referral to adult safeguarding? Did he have capacity to leave the hospital? What if he refused all help?

I also knew that while highlighting this patient to my consultant and, with the patient's consent, the social worker or the adult safeguarding lead, I wouldn't receive the necessary guidance, advice and expertise that this complex case demanded.

\section{Modern slavery}

Modern slavery is an abominable global trade in men, women and children for the purpose of forced labour, sexual exploitation and forced marriage, domestic servitude and forced criminal activities. More human beings than at any other time in history are being treated as commodities for financial gain, frequently moved from place to place, within and across borders. Sometimes forced, sometimes lured, manipulated or deceived by the promise of work, a better future or even love. On any given day, 40.3 million people are estimated to be living as slaves across the globe. ${ }^{1}$ In 2012, the Home Office estimated between 10,000-13,000 victims of modern slavery were living and working in the UK, ${ }^{2}$ however the Global Slavery Index suggests this is closer to 136,000 - every two per 1,000 individuals. ${ }^{3}$

Labour exploitation remains the most common form of exploitation, both globally and within the UK. ${ }^{1,4}$ In 2018 , nearly nine in 10 victims of forced labour identified in the UK were male, according to data from the National Referral Mechanism (NRM), a framework devised by government to identify, support and protect victims. ${ }^{4}$ Most were from the UK itself, Vietnam, Albania, Sudan and Romania. ${ }^{4}$

Although forced labour is poorly understood, trends and patterns of exploitation exist. Commonly, male victims are offered an employment package through an agency, including work, accommodation and transport. Once at the destination the individual's identification documents and passport are removed for 'safe keeping', they may be lumbered with a large, unpayable debt. They are then forced to work under the threat of punishment or by witnessing the punishment of others, threatened with denunciation to the authorities and deportation. Often they are paid below minimum wage or not at all, without employment contracts, in unsafe working conditions for exhausting hours. ${ }^{5}$ UKbased industries in which trafficked individuals have been forced to work, include farming and agriculture, construction, car washes, hospitality industries and nail salons. ${ }^{5}$

My story in the emergency department is not isolated, nor is it rare. Victims and survivors of modern slavery are presenting in healthcare settings. Multiple retrospective studies from around the world consistently suggest a significant proportion of survivors had accessed healthcare, while in slavery. ${ }^{6}$ In 2015, 782 NHS healthcare professionals were surveyed. One in eight reported previous contact with a patient that they knew or strongly suspected of having been trafficked. Within a maternity setting, this was one in five. Yet healthcare professionals felt ill-equipped to know how to respond. Eight in 10 felt they hadn't had enough training and $95 \%$ were unaware of the scale of trafficking in the UK. ${ }^{7}$

\section{Problems with the healthcare response}

Using the case of my patient in the emergency department, we can observe where the gaps lie within the healthcare service that prevent vulnerable and potentially trafficked patients being identified and supported.

Firstly, the culture of safeguarding is not prioritised. This starts back at the beginning of our education. As a medical student, I received an hour or two of my 5-year degree, dedicated to domestic abuse and violence. A reality affecting one in four women and one in seven men with significant sequelae on physical, mental and sexual health. ${ }^{8}$ If a disease had that prevalence and impact it would take a dramatically larger proportion of our time, education and resources. Once qualified, the tick-box nature of ongoing safeguarding training, with online modules or didactic training is enough to disengage even the most passionate of healthcare professionals. The default advice to 'discuss with your safeguarding lead' consistently ignores the importance of knowing what to do when a patient is sitting in front of you. How do I remove the accompanying person without raising their alarm? How do I get an independent translator, when the accompanying person is insisting on translating on the patient's behalf? What sort of questions can I ask? What kind of questions should I avoid due to the risk of re-traumatisation?

The lack of emphasis on softer communication and safeguarding skills throughout all levels of medical education, inevitably impacts on how safeguarding is prioritised in practice as qualified healthcare professionals. When combined with the environmental and situational pressures in the emergency department, for example, it is not surprising that patients are not receiving the care they need.

Secondly, without the emphasis and training background, healthcare professionals are not given the opportunities to practice applying complex consultation skills in a safe, educational environment. Contrary to this, when learning to manage a deteriorating patient, training frequently involves simulation; a safe opportunity to practice $A B C D E$, while receiving detailed feedback. This should be the case with safeguarding and communication skills. Confidence to know when and how to ask, 'Is anyone making you do anything that you don't want to do?' doesn't always come naturally or fluently. As with learning how to take a medical history, practice makes perfect.

Thirdly, healthcare professionals need to be aware of and trust the systems in place that will be responsible for their patient once referred. In the UK, a potential victim of modern slavery must be referred into the NRM, in order to access the safehouse, financial and legal support available from the government. The NRM has several serious flaws that can impact on an individual's short- and long-term recovery, and without measuring outcomes, it is unclear whether it even reduces the risk of re-trafficking. ${ }^{9}$ Healthcare 
professionals are in a unique situation therefore, being a service provider most likely to encounter potentially trafficking people, while retaining independence to offer support in the person's best interest.

In spite of this, general awareness of the existence of the NRM or alternative survivor support options even among safeguarding teams is very low. Thus identified patients are not being given the care they need, or even the opportunity to make an informed decision about where and how they would like help.

\section{Recommendations}

In order to address these issues, the healthcare response needs to focus on training, service provision and advocacy.

Firstly, training: in order to shift culture and attitudes around safeguarding, effective and engaging training methods must be adopted in medical schools and throughout professional development. Trauma-informed training that focuses on equipping healthcare professionals with transferable and applicable consultation skills, with opportunities for practice, feedback and debriefing, simulation training with actors for example.

Secondly, service provision: basic guidance, protocols and advice need to be made available to all healthcare professionals who may be concerned that their patient is vulnerable to or experiencing exploitation. Safeguarding leads should be equipped to understand the complexity of modern slavery, the strengths and weaknesses of the support services available and trauma-informed care. With this, they would be better able to manage someone's acute needs, make best-interest decisions when necessary and to support adults with capacity to make informed decisions.

Thirdly, advocacy: the Modern Slavery Act 2015 has been criticised for not addressing the needs of victims and survivors, focusing heavily on legislation, criminality and convictions. Healthcare professionals with an understanding of short-, intermediate- and long-term consequences of complex trauma must be involved within all elements of the modern slavery response in order to support survivor recovery, reduced barriers to healthcare, while focusing on risk factors for vulnerability and prevention.

\section{References}

1 International Labour Organization. Global estimates of modern slavery: Forced labour and forced marriage. Geneva: ILO, 2017.

2 HM Government. Modern slavery strategy. London: The Stationery Office, 2014. https://assets.publishing.service.gov.uk/government/ uploads/system/uploads/attachment_data/file/383764/Modern_ Slavery_Strategy_FINAL_DEC2015.pdf [Accessed 30 June 2019].

3 The Minderoo Foundation. Global Slavery Index: Country data. The Minderoo Foundation, 2018. www.globalslaveryindex.org/2018/ data/country-data/united-kingdom [Accessed 23 January 2019].

4 National Crime Agency: Vulnerabilities Command / MSHTU. National referral mechanism statistics - end of year summary 2018. NCA, 2019. https://nationalcrimeagency.gov.uk/who-we-are/ publications/282-national-referral-mechanism-statistics-end-of-yearsummary-2018/file [Accessed 30 June 2019].

5 Skrivankova K. Forced labour in the United Kingdom. York: Joseph Rowntree Foundation, 2014. www.gla.gov.uk/media/1584/jrf-forcedlabour-in-the-uk.pdf [Accessed 30 June 2019].

6 Chisolm-Straker M, Baldwin S, Gaïgbé-Togbé B et al. Healthcare and human trafficking: we are seeing the unseen. J Health Care Poor Underserved 2016;27:1220-33.

7 Ross C, Dimitrova S, Howard LM et al. Human trafficking and health: a cross-sectional survey of NHS professionals' contact with victims of human trafficking. BMJ Open 2015;5:e008682.

8 Office for National Statistics. Domestic abuse: findings from the crime survey for England and Wales: year ending March 2017. Newport: ONS, 2018. www.ons.gov.uk/peoplepopulationandcommunity/crimeandjustice/articles/domesticabusefindingsfromthecrimesurveyforenglandandwales/yearendingmarch2017\#mainpoints [Accessed 30 June 2019].

9 Home Office. Review of the National Referral Mechanism for victims of human trafficking. Home Office, 2014. https://assets.publishing. service.gov.uk/government/uploads/system/uploads/attachment_ data/file/467434/Review_of_the_National_Referral_Mechanism_ for_victims_of_human_trafficking.pdf [Accessed 30 June 2019].

Address for correspondence: Dr Rosie Riley, Pitt Farm, Ford, Wiveliscombe, Somerset TA4 2RH, UK.

Email: rosieriley@vita-training.com 